\title{
Study of temperature of black holes in terms of Chandrasekhar limit
}

\author{
Dipo Mahto ${ }^{1, a}$, Brajesh Kumar Jha ${ }^{2, b}$, Krishna Murari Singh ${ }^{1, c}$, Mahendra Ram ${ }^{3, d}$ \\ ${ }^{1}$ Department of Physics, Marwari College, T.M.B.U., Bhagalpur, India \\ ${ }^{2}$ University Department of Physics, L.N.M.U. Darbhanga, India \\ ${ }^{3}$ Department of Physics, G. L. A. PG College, N.P.U. Daltanganj, India

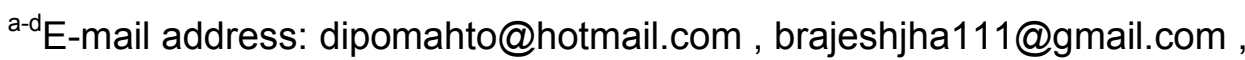 \\ kmsinghphy@gmail.com , prof.m.ram23@gmail.com
}

\begin{abstract}
Stephen Hawking gave the formula for the temperature for the black holes as $T=\frac{\hbar}{8 \pi k G M}$. In the present research article, we have converted this formula in terms of Chandrasekhar limit $\left[\mathrm{M}_{\mathrm{ch}}\right]$ and also calculated their values for different test black holes existing in XRBs and AGN. $T=3.165\left(\frac{M_{c h}}{M}\right) \times 10^{20}$ Kelvin.
\end{abstract}

Keywords: black holes; formula for the temperature; Hawking; Einstein

\section{INTRODUCTION}

A black hole is a solution of Einstein's gravitational field equations in the absence of matter that describes the space time around a gravitationally collapsed star. Its gravitational pull is so strong that even light cannot escape from it $[1,2]$.

In classical theory black holes can only absorb and not emit particles. However, it is shown that quantum mechanical effects cause black holes to create and emit particles as if they were hot bodies with temperature $\left(M_{\bullet} / M\right) \times 10^{-6} \kappa$, where $\kappa$ is surface gravity of black holes and $\mathrm{k}$ is the Boltzmann constant. This thermal emission leads to show a decrease in the mass of the black hole and to its eventual disappearance: any primordial black hole of mass less than about $10^{15}$ gram would have evaporated by now [3].

Hawking $(1974,1975)$ introduced what is now called Hawking radiation as the effective black body radiation from a black hole in terms of the 4th power of the black hole temperature and the Stefan-Boltzmann constant [3,4]. Stephan Hawking provided a theoretical argument for its existence in 1974, and sometimes also after the physicist Jacob Bekenstein who predicted that black holes should have a finite, non-zero temperature and entropy and also stated that it is proportional to the black hole area A [5].

Later on, Bardeen, Carter and Hawking performed calculations using a semi-classical approximation, putting Bekenstein conjecture on a firm basis. They established that the black 
hole temperature is proportional to its surface gravity [6]. Silva proposed intuitive derivations of the Hawking temperature and the Bekenstein-Hawking entropy of a Schwarzschild black hole [7].

Ved Prakash et al. has discussed the statistical analysis of lifetime and temperature of the black holes have been studied existing in X-ray binaries and active galactic nuclei[8]. Mehta et al. derived an expression for the variation of temperature of the black holes with respect to mass and also calculated their values of different test black holes existing only in $\mathrm{X}$-ray binaries [9].

The present research article discusses the conversion of Stephen Hawking temperature of black holes as given by formula $T=\frac{\hbar}{8 \pi k G M}$ Kelvin in terms of Chandrasekhar limit $\left[\mathrm{M}_{\mathrm{ch}}\right]$ and also calculates their values of different black holes existing in XRBs and AGN.

\section{DISCUSSION}

Silva proposed intuitive derivations of the Hawking temperature of a black hole of mass $M$ in terms of fundamental parameters is given by the following equation [7]:

$$
T=\frac{\hbar}{8 \pi k G M} \text { Kelvin }
$$

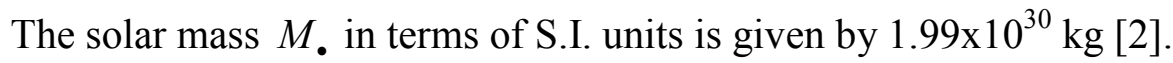

The temperature of a black hole of mass $M$ in terms of solar mass can be written by using the values of $\hbar, \mathrm{c}$, and $\mathrm{k} \mathrm{G}$ in the above equation and solving, we have

$$
T=4.557\left(\frac{M_{\bullet}}{M}\right) \times 10^{21} \text { Kelvin. }
$$

The above equation can be written as

$$
\begin{gathered}
T=4.557\left(\frac{1.44 M_{\bullet}}{1.44 M}\right) \times 10^{21} \\
T=3.165\left(\frac{1.44 M_{\bullet}}{M}\right) \times 10^{21}
\end{gathered}
$$

Now we shall convert the above expression in terms of the Chandrasekhar limit $\left(\mathrm{M}_{\mathrm{ch}}\right)$ and defined as the maximum mass of a white dwarf star, about 1.4 times the mass of the Sun. Above this mass, the gravitational pull becomes too great, and the star must collapse to a neutron star or black hole [10]. i.e.

$$
M_{c h}=1.44 M
$$

Using the equation (6) into equation (5), we have 


$$
T=3.165\left(\frac{M_{c h}}{M}\right) \times 10^{21} \text { Kelvin }
$$

\section{DATA IN SUPPORT OF MASS OF BLACK HOLES}

There are two categories of black holes classified on the basis of their masses clearly very distinct from each other, with very different masses $M \sim 5-20 M_{\odot}$ for stellar - mass black holes in X-ray binaries and $\mathrm{M} \sim 10^{6}-10^{9.5} \mathrm{M}_{\odot}$ for super massive black holes in galactic nuclei [11-13].

The mass of spinning black holes in terms of Chandrasekhar limit corresponding to the masses $\mathrm{M} \sim 5-20 \mathrm{M}_{\odot}$ for stellar - mass black holes in X-ray binaries are $3 \mathrm{M}_{\mathrm{ch}}$ to $13 \mathrm{M}_{\mathrm{ch}}$ and for the masses $\mathrm{M} \sim 10^{6}-10^{9.5} \mathrm{M}_{\odot}$ in super massive black holes in the active galactic nuclei are $1 \times 10^{6} \mathrm{M}_{\text {ch }}$ to $3 \times 10^{9} \mathrm{M}_{\text {ch }}$.

On the basis of eqn(7), we have calculated temperature of black hole in XRBs and AGN in the Table $1 \& 2$ respectively.

Table 1. Temperature of black hole in XRBs.

\begin{tabular}{|c|c|c|c|}
\hline S. No & $\begin{array}{c}\text { Mass of black } \\
\text { holes in terms } \\
\text { of } \mathrm{M}_{\mathrm{ch}}\end{array}$ & $T=3.165\left(\frac{M_{c h}}{M}\right) \times 10^{20}$ Kelvin & $|\log \Gamma|$ \\
\hline 1 & $3 \mathrm{M}_{\mathrm{ch}}$ & $10.550 \times 10^{20}$ & 21.0232 \\
\hline 2 & $4 \mathrm{M}_{\mathrm{ch}}$ & $7.912 \times 10^{20}$ & 20.8982 \\
\hline 3 & $5 \mathrm{M}_{\mathrm{ch}}$ & $6.330 \times 10^{20}$ & 20.8014 \\
\hline 4 & $6 \mathrm{M}_{\mathrm{ch}}$ & $5.275 \times 10^{20}$ & 20.6552 \\
\hline 5 & $7 \mathrm{M}_{\mathrm{ch}}$ & $4.521 \times 10^{20}$ & 20.5972 \\
\hline 6 & $8 \mathrm{M}_{\mathrm{ch}}$ & $3.956 \times 10^{20}$ & 20.5460 \\
\hline 7 & $9 \mathrm{M}_{\mathrm{ch}}$ & $3.516 \times 10^{20}$ & 20.5003 \\
\hline 8 & $10 \mathrm{M}_{\mathrm{ch}}$ & $3.165 \times 10^{20}$ & 20.4581 \\
\hline 9 & $11 \mathrm{M}_{\mathrm{ch}}$ & $2.872 \times 10^{20}$ & 20.4211 \\
\hline 10 & $12 \mathrm{M}_{\mathrm{ch}}$ & $2.637 \times 10^{20}$ & 2.3863 \\
\hline 11 & $13 \mathrm{M}_{\mathrm{ch}}$ & $2.434 \times 10^{20}$ & \\
\hline
\end{tabular}


Table 2. Temperature of black hole in AGN.

\begin{tabular}{|c|c|c|c|c|}
\hline S. No. & $\begin{array}{c}\text { Mass of black } \\
\text { holes in terms of } \\
\text { Chandrasekhar } \\
\text { limit }\left(M_{\mathrm{ch}}\right) \\
\end{array}$ & $\log M_{c h}$ & $T=3.165\left(\frac{M_{c h}}{M}\right) \times 10^{20}$ kelvin & $\log \mathrm{T}$ \\
\hline 1 & $1 \times 10^{6} \mathrm{M}_{\mathrm{ch}}$ & 6.0000 & $3.165 \times 10^{14}$ & 14.5003 \\
\hline 2 & $2 \times 10^{6} \mathrm{M}_{\mathrm{ch}}$ & 6.3010 & $1.580 \times 10^{14}$ & 14.1986 \\
\hline 3 & $3 \times 10^{6} \mathrm{M}_{\mathrm{ch}}$ & 6.4771 & $1.055 \times 10^{14}$ & 14.0232 \\
\hline 4 & $4 \times 10^{6} \mathrm{M}_{\mathrm{ch}}$ & 6.6020 & $0.791 \times 10^{14}$ & 13.8990 \\
\hline 5 & $5 \times 10^{6} \mathrm{M}_{\mathrm{ch}}$ & 6.6989 & $0.633 \times 10^{14}$ & 13.8014 \\
\hline 6 & $6 \times 10^{6} \mathrm{M}_{\mathrm{ch}}$ & 6.7781 & $0.527 \times 10^{14}$ & 13.7218 \\
\hline 7 & $7 \times 10^{6} \mathrm{M}_{\mathrm{ch}}$ & 6.8450 & $0.452 \times 10^{14}$ & 13.6551 \\
\hline 8 & $8 \times 10^{6} \mathrm{M}_{\mathrm{ch}}$ & 6.9030 & $0.395 \times 10^{14}$ & 13.5965 \\
\hline 9 & $9 \times 10^{6} \mathrm{M}_{\mathrm{ch}}$ & 6.9542 & $0.351 \times 10^{14}$ & 13.5453 \\
\hline 10 & $1 \times 10^{7} \mathrm{M}_{\mathrm{ch}}$ & 7.0000 & $3.165 \times 10^{13}$ & 13.5003 \\
\hline 11 & $2 \times 10^{7} \mathrm{M}_{\mathrm{ch}}$ & 7.3010 & $1.580 \times 10^{13}$ & 13.1986 \\
\hline 12 & $3 \times 10^{7} \mathrm{M}_{\mathrm{ch}}$ & 7.4771 & $1.055 \times 10^{13}$ & 13.0232 \\
\hline 13 & $4 \times 10^{7} \mathrm{M}_{\mathrm{ch}}$ & 7.6020 & $0.791 \times 10^{13}$ & 12.8990 \\
\hline 14 & $5 \times 10^{7} \mathrm{M}_{\mathrm{ch}}$ & 7.6989 & $0.633 \times 10^{13}$ & 12.8014 \\
\hline 15 & $6 \times 10^{7} \mathrm{M}_{\mathrm{ch}}$ & 7.7781 & $0.527 \times 10^{13}$ & 12.7218 \\
\hline 16 & $7 \times 10^{7} \mathrm{M}_{\mathrm{ch}}$ & 7.8450 & $0.452 \times 10^{13}$ & 12.6551 \\
\hline 17 & $8 \times 10^{7} \mathrm{M}_{\mathrm{ch}}$ & 7.9030 & $0.395 \times 10^{13}$ & 12.5965 \\
\hline 18 & $9 \times 10^{7} \mathrm{M}_{\mathrm{ch}}$ & 7.9542 & $0.351 \times 10^{13}$ & 12.5453 \\
\hline 19 & $1 \times 10^{8} \mathrm{M}_{\mathrm{ch}}$ & 8.0000 & $3.165 \times 10^{12}$ & 12.5003 \\
\hline 20 & $2 \times 10^{8} \mathrm{M}_{\mathrm{ch}}$ & 8.3010 & $1.580 \times 10^{12}$ & 12.1986 \\
\hline 21 & $3 \times 10^{8} \mathrm{M}_{\mathrm{ch}}$ & 8.4771 & $1.055 \times 10^{12}$ & 12.0232 \\
\hline 22 & $4 \times 10^{8} \mathrm{M}_{\mathrm{ch}}$ & 8.6020 & $0.791 \times 10^{12}$ & 11.8990 \\
\hline
\end{tabular}




\begin{tabular}{|c|c|c|c|c|}
\hline 23 & $5 \times 10^{8} \mathrm{M}_{\mathrm{ch}}$ & 8.6989 & $0.633 \times 10^{12}$ & 11.8014 \\
\hline 24 & $6 \times 10^{8} \mathrm{M}_{\mathrm{ch}}$ & 8.7781 & $0.527 \times 10^{12}$ & 11.7218 \\
\hline 25 & $7 \times 10^{8} \mathrm{M}_{\mathrm{ch}}$ & 8.8450 & $0.452 \times 10^{12}$ & 11.6551 \\
\hline 26 & $8 \times 10^{8} \mathrm{M}_{\mathrm{ch}}$ & 8.9030 & $0.395 \times 10^{12}$ & 11.5965 \\
\hline 27 & $9 \times 10^{8} \mathrm{M}_{\mathrm{ch}}$ & 8.9542 & $0.351 \times 10^{12}$ & 11.5453 \\
\hline 28 & $1 \times 10^{9} \mathrm{M}_{\mathrm{ch}}$ & 9.0000 & $3.165 \times 10^{11}$ & 11.5003 \\
\hline 29 & $2 \times 10^{9} \mathrm{M}_{\mathrm{ch}}$ & 9.3010 & $1.580 \times 10^{11}$ & 11.1986 \\
\hline 30 & $3 \times 10^{9} \mathrm{M}_{\mathrm{ch}}$ & 9.4771 & $1.055 \times 10^{11}$ & 11.0232 \\
\hline
\end{tabular}

\section{Graph 1:}

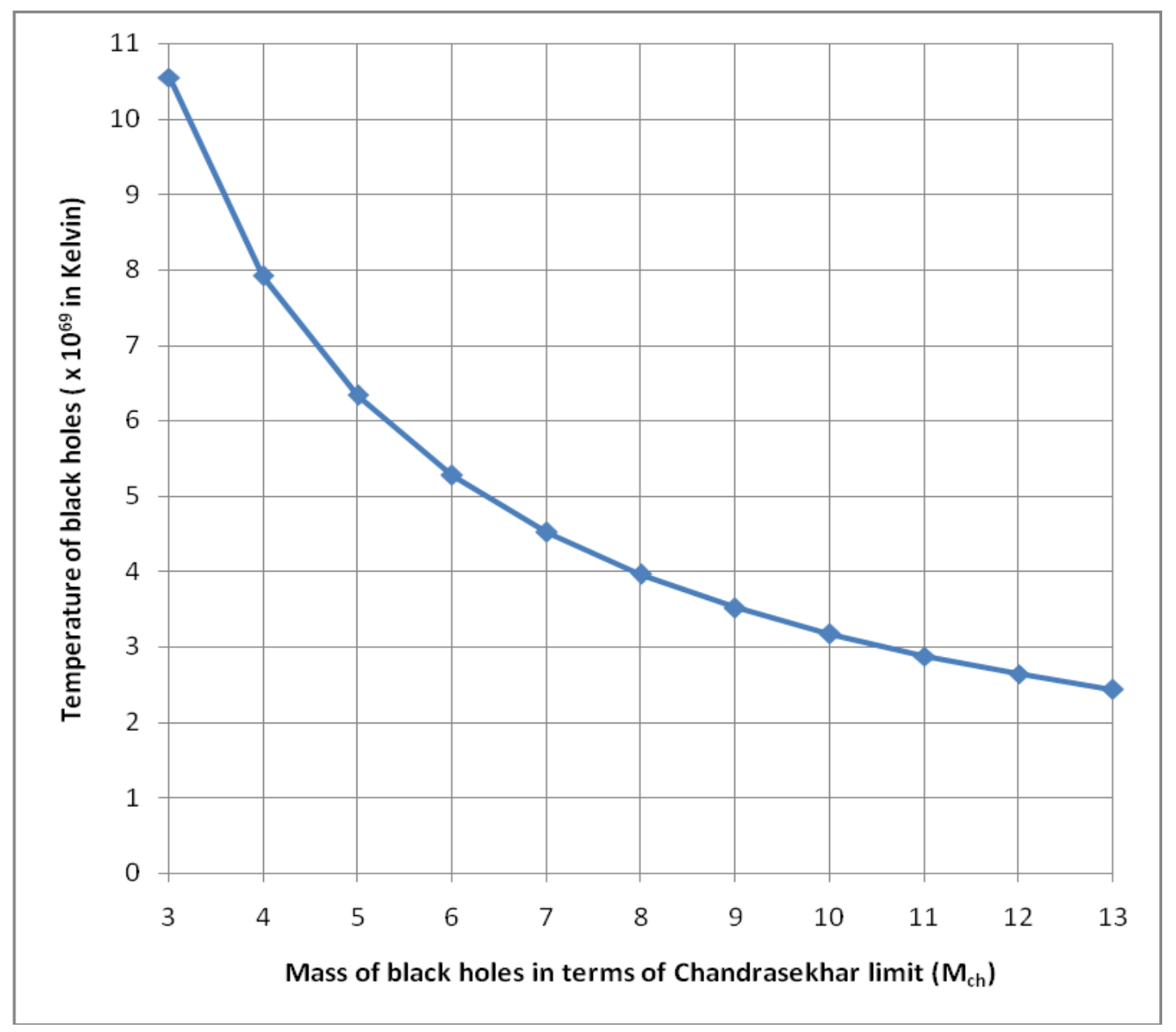

Fig. 1. The graph plotted bet ${ }^{\mathrm{n}}$ the mass of black holes and their corresponding temperature in XRBs. 


\section{Graph 2:}

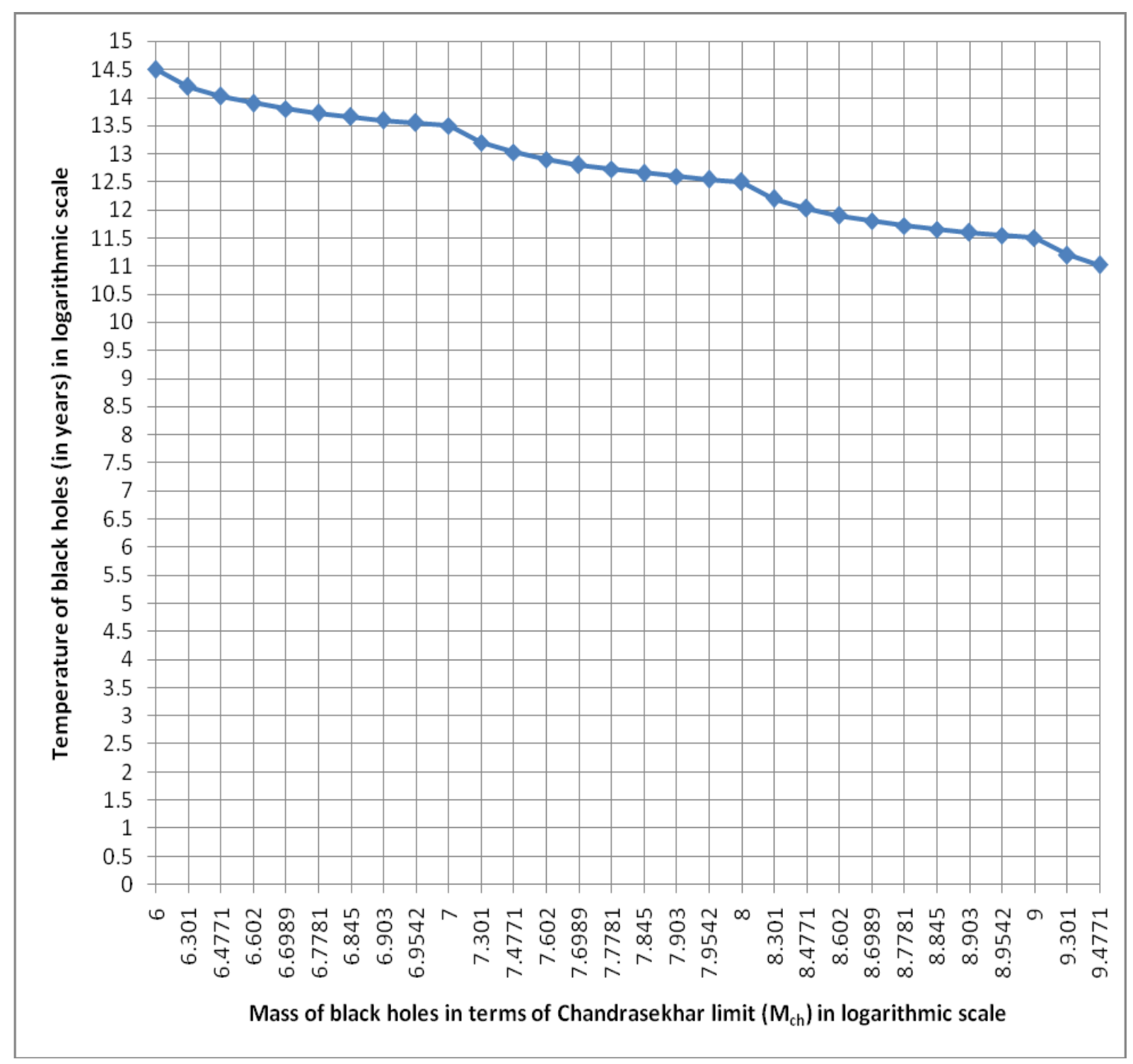

Fig. 2. The graph plotted bet ${ }^{\mathrm{n}}$ the mass of black holes and their corresponding temperature in AGN.

\section{RESULTS AND DISCUSSION}

In the present work, we have converted the formula for temperature of black holes in terms of Chandrasekhar limit and calculated their values for the different test black holes in $\mathrm{X}$-ray binaries (XRBs) and Active galactic nuclei (AGN). To discuss the temperature of black holes with the mass $\left(\mathrm{M}_{\mathrm{ch}}\right)$, graphs have been plotted between:

(i) the mass of different test black holes $\left(\mathrm{M}_{\mathrm{ch}}\right)$ and their corresponding values of temperature in XRBs (Fig. 1 )

(ii) mass of different test black holes $\left(\mathrm{M}_{\mathrm{ch}}\right)$ and their corresponding values of temperature using logarithmic scale in AGN (Fig. 2) 
The Figure 1 shows that the temperature of black holes decreases with the increase of the mass of the different test black holes in XRBs, while in the Figure 2, the variation of temperature of black holes with the mass in the case of AGN is not be the same to that of XRBs, but differing in the some manner.

In this case, the temperature of black holes decreases with increase in the mass of black holes. From the graph, it is clear that the nature of variation of temperature of black holes with their corresponding mass in AGN is repeated in the same manner after certain different mass. These certain different masses are $10^{6} \mathrm{M}_{\mathrm{ch}}, 10^{7} \mathrm{M}_{\mathrm{ch}}, 10^{8} \mathrm{M}_{\mathrm{ch}}$ and $10^{9} \mathrm{M}_{\mathrm{ch}}$.

These data of masses of black holes are in the multiple of ten (10) and hence these masses have special characters.

\section{CONCLUSIONS}

During the study of present research work, we can draw the following conclusions such as:

(i) The temperature of black holes decreases with the increase in the mass of the different test black holes in XRBs showing that heavier mass of black hole has smaller temperature and vice-versa.

(ii) The nature of variation of the temperature of black holes with their corresponding mass in AGN is repeated in the same manner after certain different mass.

(iii) The mass of black holes in the multiple of ten (10) has special character.

\section{References}

[1] Dabholkar A., Current Science 89(12) (2005) 2054-2063.

[2] Mahto D., Prakash V., Prasad U., Singh B. K., Singh K. M., Astrophysics Space Science 343(1) (2013) 153-159.

[3] Hawking S. W., Commun Math. Phys. 43 (1975) 199-220.

[4] Hawking S.W., Nature 248 (1974) 30-31.

[5] Hawking S. W., Bantam Books (1988). ISBN 0-553-38016-8.

[6] Bardeen J. M., Carter B., Hawking S. W., Commun. Math. Phy. 31(2) (1973) 161-170.

[7] Silva P. R., General Relativity and Quantum Cosmology, 2006.

[8] Prakash V., Mahto D., Kumar A. \& Das B. K., American Journal of Theoretical and Applied Statistics 2(6) (2013) 228-232.

[9] Mehta R. N., Prasad U., Kumar A., Mahto D, International Journal of Astrophysics and Space Science 1(4) 2013) 61-63.

[10] Carroll S., Ph.D., Cal Tech, 2007, The Teaching Company, Guidebook Part 2, page 44, Accessed Oct. 7, 2013.

[11] R. Narayan, New Journal Physics 7(1) (2005) 1-31. 
[12] Dipo Mahto, Tanveer Ali Khan, Ashok Kumar, Raj Kumar Sah, International Letters of Chemistry, Physics and Astronomy 4 (2014) 12-19.

[13] Dipo Mahto, Amresh Kumar Singh, Kumari Vineeta, Ashok Kumar, International Letters of Chemistry, Physics and Astronomy 13(2) (2014) 95-103. 